\title{
Superconducting boron allotropes
}

\author{
Shoutao Zhang $\odot,{ }^{1}$ Xin Du, ${ }^{1}$ Jianyan Lin $\odot,{ }^{1}$ Aitor Bergara $\odot, 3,4,5, *$ Xin Chen, ${ }^{2}$ Xiaobing Liu,,${ }^{2, \dagger}$ \\ Xiaohua Zhang, ${ }^{1}$ and Guochun Yang $\oplus^{1, \ddagger}$ \\ ${ }^{1}$ Centre for Advanced Optoelectronic Functional Materials Research and Laboratory for UV Light-Emitting Materials and Technology of \\ Ministry of Education, Northeast Normal University, Changchun 130024, China \\ ${ }^{2}$ Laboratory of High Pressure Physics and Material Science, School of Physics and Physical Engineering, \\ Qufu Normal University, Qufu, Shandong Province 273165, China \\ ${ }^{3}$ Departamento de Física de la Materia Condensada, Universidad del País Vasco-Euskal Herriko Unibertsitatea, \\ UPV/EHU, 48080 Bilbao, Spain \\ ${ }^{4}$ Donostia International Physics Center (DIPC), 20018 Donostia, Spain \\ ${ }^{5}$ Centro de Física de Materiales CFM, Centro Mixto CSIC-UPV/EHU, 20018 Donostia, Spain
}

(Received 27 February 2020; revised manuscript received 11 April 2020; accepted 20 April 2020; published 11 May 2020)

\begin{abstract}
The search for elemental allotropes is an active research field to get unusual structures with unique properties. The removal of metal atoms from pressure-induced stable binary compounds has become a useful method for obtaining elemental allotropes with interesting properties that otherwise would not be accessible at ambient pressure. Although three-dimensional boron allotropes have been studied extensively, none of those found so far are superconducting at ambient pressure. Here we propose that $\mathrm{NaB}_{4}$ and $\mathrm{Na}_{2} \mathrm{~B}_{17}$ can be used as precursors to achieve superconducting boron allotropes at ambient pressure. First-principle swarm-intelligence structure search calculations identify several novel sodium borides (e.g., $\mathrm{Na}_{3} \mathrm{~B}_{2}, \mathrm{Na}_{2} \mathrm{~B}_{3}, \mathrm{NaB}_{4}$, and $\mathrm{Na}_{2} \mathrm{~B}_{17}$ ) under high pressure. Interestingly, the $\mathrm{B}$ atoms in $14 / \mathrm{mmm} \mathrm{NaB}$ and $P m \mathrm{Na}_{2} \mathrm{~B}_{17}$ form three-dimensional frameworks with open channels, where $\mathrm{Na}$ atoms are located. After the removal of $\mathrm{Na}$ atoms, two hitherto unknown boron allotropes, named as $I 4 / \mathrm{mmm} \mathrm{B}_{4}$ and $P m \mathrm{~B}_{17}$, are stable at ambient pressure. They are metallic with superconducting critical temperatures of 19.8 and $15.4 \mathrm{~K}$, respectively, becoming the highest ones among bulk boron allotropes. In addition, considering their predicted Vickers hardness of 27.3 and $26.8 \mathrm{GPa}$, they are also potential hard materials.
\end{abstract}

DOI: 10.1103/PhysRevB.101.174507

\section{INTRODUCTION}

The preparation of high-temperature superconductors is of great importance for fundamental research and practical applications [1-6]. Recently discovered pressure-induced stable H-rich compounds (e.g., $\mathrm{H}_{3} \mathrm{~S}$ [7] and $\mathrm{LaH}_{10}$ [8,9]) broke the superconducting transition temperature record of $164 \mathrm{~K}$ in cuprates [10]. Notably, theoretical predictions play a leading role in accelerating these innovative discoveries [11-13]. Very recently, room-temperature or even higher-temperature superconductivity has been predicted through doping lithium into $\mathrm{MgH}_{16}$, forming a novel ternary compound, $\mathrm{Li}_{2} \mathrm{MgH}_{16}$ [14]. However, a common characteristic of these hydrides is that their superconductivity emerges at more than one million times the atmospheric pressure. Therefore, the search for stable or metastable superconducting materials at ambient pressure is highly needed for practical application [15].

Some of pressure-induced stable compounds are recoverable to ambient pressure with interesting properties, especially for light mass elements, such as boron, carbon, and nitrogen.

\footnotetext{
*Corresponding author: a.bergara@ehu.eus

${ }^{\dagger}$ Corresponding author: xiaobing.phy@qfnu.edu.cn

${ }^{\ddagger}$ Corresponding author: yanggc468@ nenu.edu.cn
}

They can be synthesized, since pressure allows them to overcome the required reaction barriers to be formed. For example, well-known superhard materials, such as $\mathrm{BC}_{3}[16]$ and diamond [17], are synthesized at high pressures and become metastable at ambient pressure. On the other hand, pressureinduced stable binary compounds are also used as precursors to achieve elemental allotropes. For instance, two silicon allotropes, $\mathrm{Si}_{24}$ [18] and $P 6 / m-\mathrm{Si}_{6}$ [19], are obtained through a two-step synthesis methodology, removing the $\mathrm{Na}$ atoms from the pressure-induced stable $\mathrm{Na}_{4} \mathrm{Si}_{24}$ and $P 6 / m-\mathrm{NaSi}_{6}$ via a thermal "degassing" process. More interestingly, $\mathrm{Si}_{24}$ has a proper band gap of $1.3 \mathrm{eV}$, showing broad applications as photovoltaic cells [18]. $P 6 / m-\mathrm{Si}_{6}$ becomes a superconductor with a critical transition temperature of $12 \mathrm{~K}$ at ambient pressure [19]. In both structures the $\mathrm{Si}$ atoms form a threedimensional frame with open channels, where the $\mathrm{Na}$ atoms are located, which makes it easy for them to escape.

The study of boron allotropes has attracted great attention because of their electron deficiency, structural complexity, and unusual bonding pattern [20,21]. Up to now, at least 14 boron allotropes have been reported [22]. Most of them consist of $B_{12}$ icosahedra [23]. However, the arrangement of the icosahedra strongly modifies their electronic properties. For instance, $I 2_{1} 2_{1} 2_{1}-\mathrm{B}_{60}$, where $\mathrm{B}_{12}$ icosahedra are linked by helical boron chains, is a metal, whereas Pnma- $\mathrm{B}_{60}$, in 
which $\mathrm{B}_{12}$ icosahedra are interconnected by two-atom wide B ribbons, is a semimetal [24]. After compression, boron allotropes undergo complex structural transitions, caused by the appearance of novel boron units, which also modify their electronic properties [23,25-29]. So far, no bulk superconducting boron allotrope has been found at ambient pressure.

Having in mind that pressure can stabilize unusual stoichiometric compounds [30-32] and that known sodium borides consist of covalent $B$ frames [33-35], we consider the binary Na-B system to explore stable B-rich Na-B compounds with open channel frames, then to obtain metastable boron allotropes after releasing $\mathrm{Na}$ atoms. In this work, first-principle swarm-intelligence structural search method was employed to identify stable Na-B compounds at high pressures. Besides reproducing the known Na-B compounds (e.g., $\mathrm{Na}_{3} \mathrm{~B}_{20}$ [33] and $\mathrm{NaB}_{15}$ [35]) at ambient pressure, four new Na-B compositions (e.g., $\mathrm{Na}_{3} \mathrm{~B}_{2}, \mathrm{Na}_{2} \mathrm{~B}_{3}, \mathrm{NaB}_{4}$, and $\mathrm{Na}_{2} \mathrm{~B}_{17}$ ) become stable under high pressure. As expected, two metastable $14 / \mathrm{mmm}_{4}$ and $P m \mathrm{~B}_{17}$ allotropes are obtained from $I 4 / \mathrm{mmm} \mathrm{NaB}_{4}$ and Pm $\mathrm{Na}_{2} \mathrm{~B}_{17}$ precursors. Interestingly, these new compounds are predicted to be highly stable and even superconductors, becoming the first superconducting bulk boron allotropes at ambient pressure.

\section{COMPUTATIONAL METHODS}

To explore the stable Na-B phases under high pressure, we employ a swarm-intelligence based CALYPSO structure search method [36,37], which can find stable structures just depending on the chemical compositions. The geometry optimization and calculations of the electronic properties are performed with the Vienna $a b$ initio simulation package (VASP) code [38] within the framework of density functional theory (DFT) $[39,40]$. The all-electron projector augmentedwave (PAW) [41] pseudopotentials with $2 s^{2} 2 p^{6} 3 s^{1}$ and $2 s^{2} 2 p^{1}$ valence electrons for $\mathrm{Na}$ and $\mathrm{B}$ atoms, respectively, are used to describe the interactions between electrons and ions. The full-potential all-electron calculations of the equation of states for $\mathrm{NaB}_{4}$ compound were performed with the full-potential linearized augmented plane-wave method as implemented in the WIEN2K code [42] to examine the validity of the selected PAW pseudopotentials under high pressure (Fig. S0). The Perdew-Burke-Ernzerhof (PBE) [43] functional within the generalized gradient approximation (GGA) [44] is used to account for the exchange-correlation energy. A cutoff energy of $800 \mathrm{eV}$ and a Monkhorst-Pack [45] $k$-point grid with a reciprocal space resolution of $2 \pi \times 0.03 \AA^{-1}$ in the Brillouin zone yield an excellent convergence for the Gibbs free energy. Electron-phonon coupling calculations within the density functional perturbation theory and the plane-wave pseudopotential method with ultrasoft pseudopotentials are implemented in the QUANTUM ESPRESSO package [46]. More detailed illustrations of structure search and computational details can be found in the Supplemental Material [47].

\section{RESULTS AND DISCUSSION}

\section{A. Phase stability}

Here we mainly focused on B-rich Na-B compounds, which have a tendency to form three-dimensional B frames.

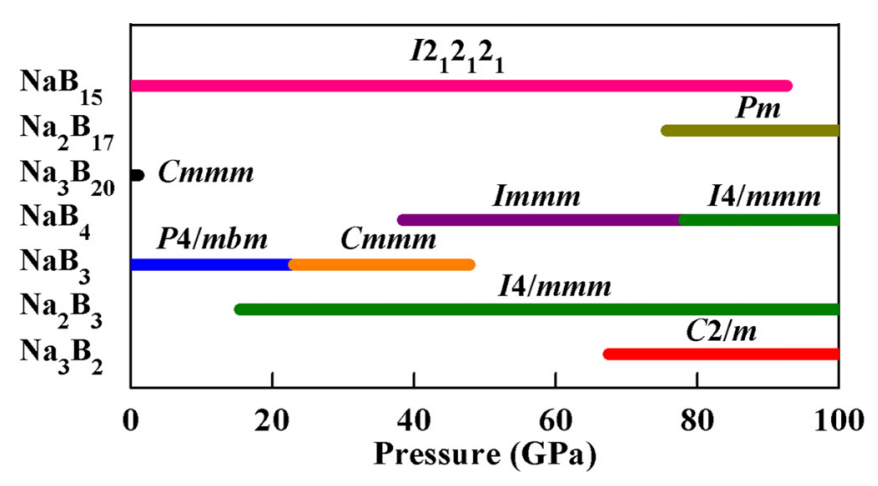

FIG. 1. Pressure-composition phase stability diagram of the NaB system between $1 \mathrm{~atm}$ and $100 \mathrm{GPa}$.

We conduct an extensive structure search on Na-B compounds with a variety of $\mathrm{Na}_{m} \mathrm{~B}_{n}(m=1, n=1-20 ; m=2, n=1,3$, $5,7,9,11,13,15,17,19 ; m=3, n=2,20)$ compositions at $0 \mathrm{~K}$ and the following pressures, $1 \mathrm{~atm}$ and 10, 25, 50, and 100 GPa. The relative thermodynamic stabilities of the considered $\mathrm{Na}_{m} \mathrm{~B}_{n}$ compounds are shown in the convex hull diagram [48] (Fig. S1). The thermodynamic stable phases sitting at the solid line are denoted by solid spheres, whereas the metastable phases, represented by open spheres, decompose into elemental $\mathrm{Na}[49,50]$ and $\mathrm{B}[23]$ solids or other $\mathrm{Na}_{m} \mathrm{~B}_{n}$ phases. At ambient pressure, the already known $\mathrm{Cmmm} \mathrm{Na}_{3} \mathrm{~B}_{20}$ and $I 22_{1} 2_{1} \mathrm{NaB}_{15}$ are reproduced in our structure search [35]. As can be seen in Fig. $1, I 22_{1} 2_{1} 2_{1} \mathrm{NaB}_{15}$ shows a much larger stable pressure range than $\mathrm{Cmmm} \mathrm{Na}_{3} \mathrm{~B}_{20}$. On the other hand, the predicted phases and phase transition pressures for $\mathrm{NaB}_{3}$ are in excellent agreement with the results of Zhou et al. (Fig. S2) [34]. All these results demonstrate that our adopted structure search method and pseudopotentials are suitable for the Na-B system.

At higher pressures, several new B-rich stoichiometries (i.e., $\mathrm{Na}_{2} \mathrm{~B}_{3}, \mathrm{NaB}_{4}$, and $\mathrm{Na}_{2} \mathrm{~B}_{17}$ ) emerge on the convex hull (Fig. S1). Their stable pressure ranges are shown in Fig. 1. In more detail, $I 4 / \mathrm{mmm} \mathrm{Na}_{2} \mathrm{~B}_{3}$ is predicted to be stable above 15.4 GPa. For $\mathrm{NaB}_{4}$, there are two stable phases in the pressure range considered: Immm $\mathrm{NaB}_{4}$ becomes stable at 38.4 $\mathrm{GPa}$ and then transforms into $I 4 / \mathrm{mmm} \mathrm{NaB}_{4}$ above $78.1 \mathrm{GPa}$. $\mathrm{Na}_{2} \mathrm{~B}_{17}$, with a higher $\mathrm{B}$ content, stabilizes above $75.6 \mathrm{GPa}$. Na-rich $C 2 / m \mathrm{Na}_{3} \mathrm{~B}_{2}$ begins to be stable above $67.5 \mathrm{GPa}$. For compounds containing light elements, zero-point energy (ZPE) potentially has a large contribution to the total Gibbs free energy, which might influence their relative stability [51-53]. However, the inclusion of ZPE for the predicted sodium borides at the selected pressure of $100 \mathrm{GPa}$ did not change their relative stabilities (Fig. S3). Phonon spectra calculations $[54,55]$ demonstrate that all $\mathrm{Na}_{m} \mathrm{~B}_{n}$ compounds are dynamically stable, with no imaginary frequency modes at any high-symmetry direction in the whole Brillouin zone (Fig. S4).

\section{B. Crystal structures}

We mainly focus on the phases of $\mathrm{NaB}_{4}$ and $\mathrm{Na}_{2} \mathrm{~B}_{17}$, consisting of three-dimensional $\mathrm{B}$ frames. The structures and electron properties of the other predicted $\mathrm{Na}_{3} \mathrm{~B}_{2}$ and $\mathrm{Na}_{2} \mathrm{~B}_{3}$ can be found in Figs. S5 and S6. The low-pressure $\mathrm{NaB}_{4}$ 
(a) Immm $\mathrm{NaB}_{4}$

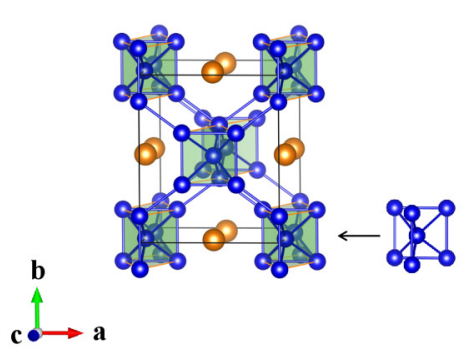

(d) I4/mmm $\mathrm{NaB}_{4}$

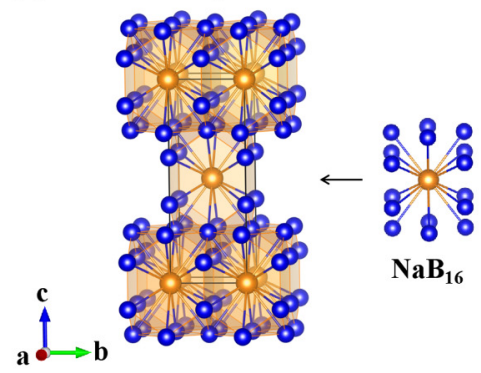

(g) $14 / \mathrm{mmm} \mathrm{B}_{4}$

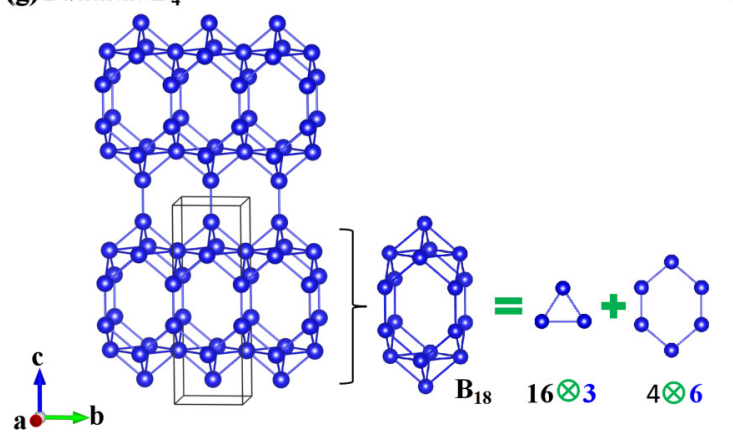

(b) Immm $\mathrm{NaB}_{4}$

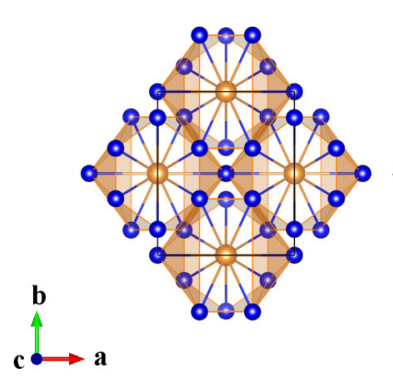

(e) $P \boldsymbol{m ~ N a} \mathbf{N a}_{17}$ (c) $14 / \mathrm{mmm} \mathrm{NaB}_{4}$

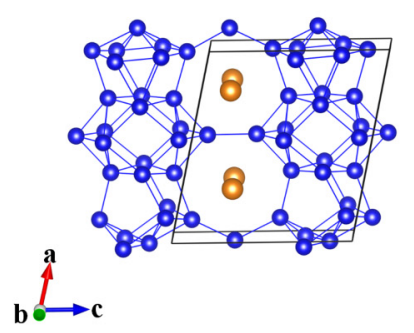

(h) $P m B_{17}$
- Na

- B

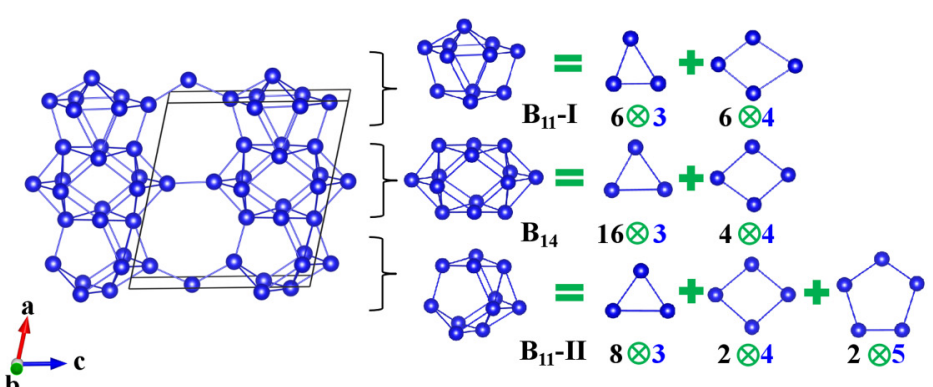

FIG. 2. Crystal structures of predicted Na-B compounds and B allotropes. (a) Immm $\mathrm{NaB}_{4}$ with $\mathrm{B}_{7}$ pentahedra at $50 \mathrm{GPa}$. (b) Immm NaB with $\mathrm{NaB}_{12}$ units at $50 \mathrm{GPa}$. (c) $I 4 / \mathrm{mmm} \mathrm{NaB}_{4}$ at $100 \mathrm{GPa}$. (d) Each $\mathrm{Na}$ atom has a 16 -fold coordination with $\mathrm{B}$ in $I 4 / m m m \mathrm{NaB}_{4}$. (e) $P m \mathrm{Na}_{2} \mathrm{~B}_{17}$ at $100 \mathrm{GPa}$. (f) Each Na atom is coordinated by 18 and $19 \mathrm{~B}$ atoms in $P m \mathrm{Na}_{2} \mathrm{~B}_{17}$. (g) $I 4 / m m m \mathrm{~B}_{4}$ at 1 atm. The $18-m e m b e r \mathrm{~B}$ unit

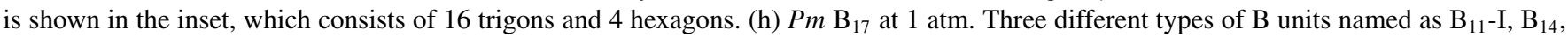
and $\mathrm{B}_{11}$-II are included in $P m \mathrm{~B}_{17}$. $\mathrm{B}_{11}$-I includes six trigons and six irregular squares. $\mathrm{B}_{14}$ is composed of 16 trigons and 4 irregular squares. $\mathrm{B}_{11}$-II contains eight trigons, two irregular squares, and two pentagons. In these structures, $\mathrm{Na}$ and $\mathrm{B}$ atoms are denoted in yellow and blue spheres, respectively.

phase is predicted to have an orthorhombic structure [space group Immm, 4 f.u. per cell, Fig. 2(a)]. There appears $\mathrm{B}_{7}$ pentahedrons with B-B bond lengths of $1.65-1.80 \AA$ at $50 \mathrm{GPa}$, and these pentahedrons make a $B$ network via edge and face sharing. Each $\mathrm{Na}$ atom is 12 -fold coordinated, with $\mathrm{B}$ forming a face-sharing $\mathrm{Na}-\mathrm{B}$ polyhedra with $\mathrm{Na}-\mathrm{B}$ bond lengths of 2.30-2.49 $\AA$ [Fig. 2(b)]. The high-pressure $\mathrm{NaB}_{4}$ phase has a tetragonal structure [space group $I 4 / \mathrm{mmm}, 4$ f.u. per cell, Fig. 2(c)], which is isostructural to $\mathrm{LiB}_{4}$ [56]. Strikingly, it shows $\mathrm{B}_{18}$ icosahedrons [Fig. 2(g)], which are interconnected to form an open-channel frame with a B-B distance of 1.70$1.96 \AA$ at $100 \mathrm{GPa}$. Notably, Na atoms sit at the center of the open channel and coordinate with $16 \mathrm{~B}$ atoms [Fig. 2(d)].

$\mathrm{Na}_{2} \mathrm{~B}_{17}$ stabilizes into a monoclinic structure [space group $P m, 1$ f.u. per cell, Fig. 2(e)], consisting of three kinds of facesharing $\mathrm{B}$ units, dubbed as $\mathrm{B}_{11}-\mathrm{I}, \mathrm{B}_{14}$, and $\mathrm{B}_{11}$-II [Fig. 2(h)]. The three $\mathrm{B}$ units interconnect with each other to form a three-dimensional framework with an open channel along the $b$ direction. The B-B bond length is $1.53-1.96 \AA$ at $100 \mathrm{GPa}$. More interestingly, $\mathrm{Na}$ atoms form linear chains located in the skeletal channels. Notably, $\mathrm{Na}$ atom in $\mathrm{Na}_{2} \mathrm{~B}_{17}$ exhibits the highest coordination numbers (up to 19) among sodium borides. Electron localization function (ELF) [57] analysis shows that $\mathrm{B}-\mathrm{B}$ bonds in three-dimensional frameworks of $\mathrm{NaB}_{4}$ and $\mathrm{Na}_{2} \mathrm{~B}_{17}$ are covalent (Fig. S7); while the Na-B bonding is weakly ionic, which is also supported by the Bader charge analysis (Table S3). To analyze the relative bond strength between Na-B and B-B bonds, the integrated crystal orbital Hamilton populations (ICOHP) are employed as implemented in the LOBSTER package $[58,59]$. The resulting ICOHPs of Na-B and B-B pairs in $I 4 / \mathrm{mmm} \mathrm{NaB}_{4}$ are -0.033 and $-3.410 \mathrm{eV} /$ pair, respectively, indicating that the Na-B interaction is much weaker than that of B-B. A silicon allotrope, $\mathrm{Si}_{24}$, has been obtained from $\mathrm{Na}_{4} \mathrm{Si}_{24}$ through removing $\mathrm{Na}$ 
atoms [18]. For comparison, the calculated ICOHPs of $\mathrm{Na}-\mathrm{Si}$ and $\mathrm{Si}-\mathrm{Si}$ pairs in $\mathrm{Na}_{4} \mathrm{Si}_{24}$ are -0.066 and $-2.544 \mathrm{eV} /$ pair, respectively. This indicates that the Na-B interaction in $\mathrm{NaB}_{4}$ is weaker than $\mathrm{Na}-\mathrm{Si}$ in $\mathrm{Na}_{4} \mathrm{Si}_{24}$, whereas the B-B bond strength is stronger than $\mathrm{Si}-\mathrm{Si}$ in $\mathrm{Na}_{4} \mathrm{Si}_{24}$. Similar results can be also seen in $P m \mathrm{Na}_{2} \mathrm{~B}_{17}$ (Table S4).

Motivated by the unique structural characteristics of $\mathrm{NaB}_{4}$ and $\mathrm{Na}_{2} \mathrm{~B}_{17}$ (covalent open $\mathrm{B}$ channels and $\mathrm{Na}$ located in the center of the open channel), we explore their energy and structural stability after removing $\mathrm{Na}$ atoms. The resulting structures, referred to as $I 4 / \mathrm{mmm} \mathrm{B}_{4}$ and $P m \mathrm{~B}_{17}$, are 0.24 and $0.25 \mathrm{eV} /$ atom higher in energy than $\alpha-\mathrm{B}_{12}$ [23] at $1 \mathrm{~atm}$ (Table S5), respectively. These are lower formation enthalpies than $P 6 / m-S_{6}(0.35 \mathrm{eV} /$ atom $)$ [19] relative to $\mathrm{Si}$ in the diamond structure at $1 \mathrm{~atm}$, indicating that they are metastable and could be synthesized at certain conditions. Their structures are comparable to the $\mathrm{B}$ sublattice in $I 4 / \mathrm{mmm} \mathrm{NaB} 4$ and $P m \mathrm{Na}_{2} \mathrm{~B}_{17}$ (their B-B covalent bonds are still maintained, Fig. S7), and are dynamically stable at ambient pressure [Figs. 3(a) and 3(b)]. Interestingly, I4/ $\mathrm{mmm} \mathrm{B}_{4}$ and Pm $\mathrm{B}_{17}$ are shown to be also mechanically stable (Table S6) [60]. In addition, we have also performed molecular dynamical simulations [61] at 1000 and $1500 \mathrm{~K}$ with a time step of $1.0 \mathrm{fs}$ to verify their thermal stability. The snapshots of the resulting structures clearly indicate that $I 4 / \mathrm{mmm} \mathrm{B}_{4}$ and $P m \mathrm{~B}_{17}$ also remain stable at these temperatures (Fig. S8), which supports their practical applicability.

Already known B allotropes at ambient and high pressures present a myriad of different structural characteristics with various interesting properties. For example, $\alpha-\mathrm{B}_{12}$, consisting of edging-sharing 20 boron trigons, is nonmetallic at ambient pressure [23]. High-pressure insulating ionic $\gamma-B_{28}$ phase consists of cationic $\mathrm{B}_{2}$ pairs and anionic icosahedral $\mathrm{B}_{12}$ containing 20 boron trigons, shows a reduction of the band gap with pressure similar to that of $\alpha-\mathrm{B}_{12}$ [23]. Metallic $\alpha$-Ga-type $\mathrm{B}$, transformed from $\alpha$ - $\mathrm{B}_{12}$ above $74 \mathrm{GPa}$, which is regarded as the modification of $\mathrm{B}_{12}$ icosahedra [27], is a superhard material. Under higher pressure ( $>375 \mathrm{GPa}$ ) $\mathrm{B}_{10}$ phase presents a linear atomic chain combined with an isosceles triangle, exhibiting a high- $T_{\mathrm{c}}$ superconductivity of $44 \mathrm{~K}$ at $400 \mathrm{GPa}$ [29]. Recently, several new metallic and hard $\mathrm{B}$ allotropes, termed as $o-\mathrm{B}_{24}(20.0 \mathrm{GPa}), c-\mathrm{B}_{56}(26.6 \mathrm{GPa})$, $m-\mathrm{B}_{16}(56.2 \mathrm{GPa})$, and $o-\mathrm{B}_{16}(60.7 \mathrm{GPa})$ from ambient to high pressure up $120 \mathrm{GPa}$ have been found, in which $o-\mathrm{B}_{24}$ has $\mathrm{B}_{12}$ icosahedra units, whereas $\mathrm{B}_{12}$ icosahedra in $c$ - $\mathrm{B}_{56}$, $m-\mathrm{B}_{16}$, and $o-\mathrm{B}_{16}$ are absent [62]. More recently, it has been reported that $t-\mathrm{B}_{106}$ at ambient conditions is just slightly less stable than $\beta$-B [63], consisting of interstitial $\mathrm{B}$ atoms and interpenetrating icosahedra and $\alpha-\mathrm{B}_{12}$ [64]. However, compared with boron units of the known $B$ allotropes at ambient and high pressures that we have briefly described above, the clathrate boron structural units in $I 4 / \mathrm{mmm} \mathrm{B}_{4}$ and $\mathrm{Pm} \mathrm{B}_{17}$ we are predicting in this work are further dismembered into smaller B subunits, which usually induce the presence of interesting properties. In the $I 4 / \mathrm{mmm} \mathrm{B}_{4}, \mathrm{~B}_{18}$ icosahedron can be seen as the edge-sharing stacking of sixteen trigons and four hexagons [Fig. 2(g)], while in $\mathrm{Pm} \mathrm{B}_{17}, \mathrm{~B}_{11}$-I has six trigons and six irregular squares. $\mathrm{B}_{14}$ includes 16 trigons and 4 irregular squares. $\mathrm{B}_{11}-\mathrm{II}$ is composed of eight trigons, two irregular squares, and two pentagons [Fig. 2(h)].
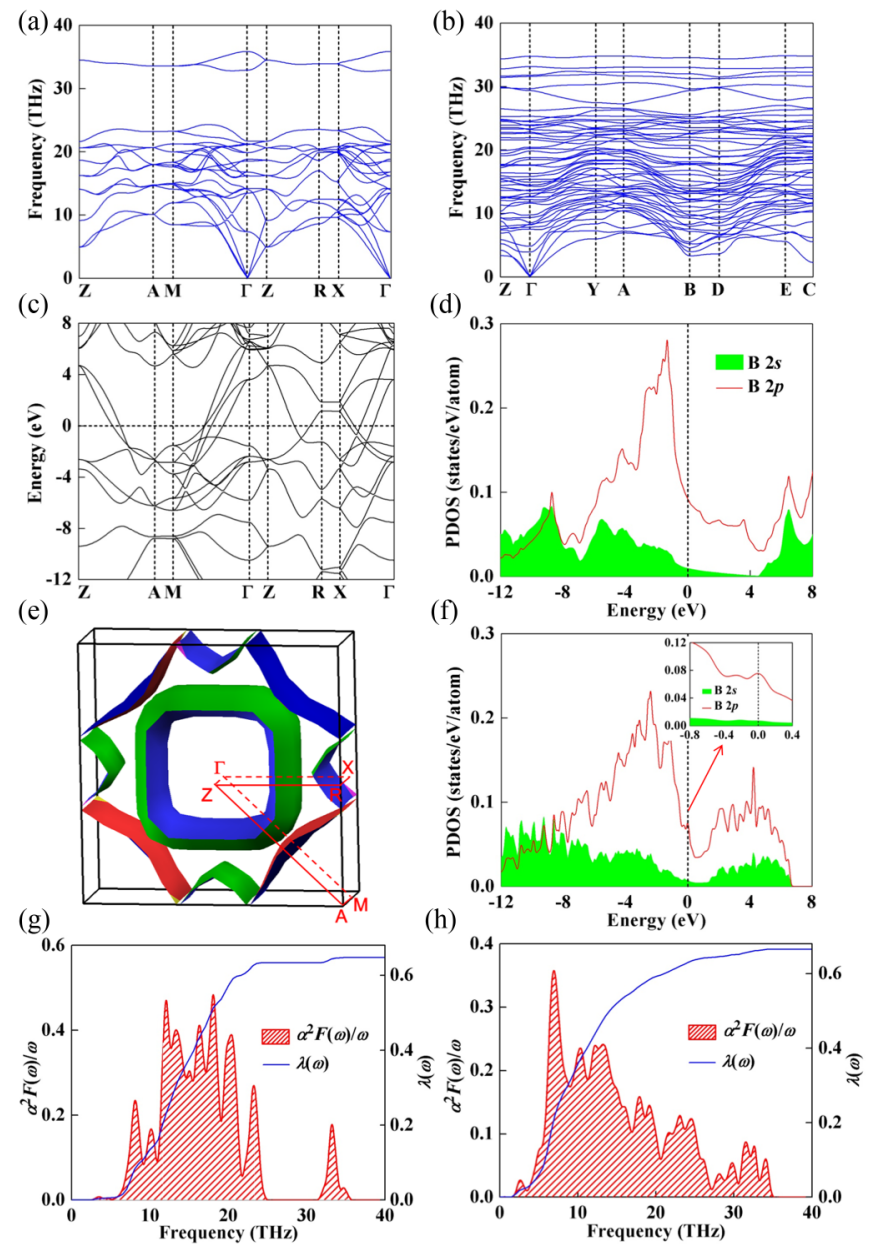

FIG. 3. Electronic and superconducting properties of I4/mmm $\mathrm{B}_{4}$ and $\mathrm{Pm} \mathrm{B}_{17}$ at 1 atm. Phonon dispersion curves of (a) $I 4 / \mathrm{mmm} \mathrm{B}_{4}$ and (b) $P m \mathrm{~B}_{17}$. (c) Electronic band structure of $I 4 / \mathrm{mmm}_{4}$. The horizontal dashed line indicates the Fermi level $E_{F}$. (d) PDOS of $I 4 / \mathrm{mmm} \mathrm{B}_{4}$. The vertical dashed line indicates the Fermi level. (e) The Fermi surface for $14 / \mathrm{mmm}_{\mathrm{B}}$. The Fermi surface associated with each band crossing the Fermi energy is displayed in Fig. S10. (f) PDOS of $P m \mathrm{~B}_{17}$. A close up of the PDOS around the van Hove singularity (vHs) near the $E_{F}$ is illustrated in the inset of (f). Eliashberg spectral function $\alpha^{2} F(\omega)$ (red area) and frequency-dependent electron-phonon coupling parameters $\lambda(\omega)$ (blue line) of (g) I4/mmm $\mathrm{B}_{4}$ and (h) $P m \mathrm{~B}_{17}$.

Considering the strong B-B covalent bonding, the threedimensional $\mathrm{B}$ framework in $I 4 / \mathrm{mmm} \mathrm{B}_{4}$ and $P m \mathrm{~B}_{17}$ and the excellent hardness in B-based compounds [65-68], we have also explored the hardness of the predicted new boron allotropes with the strain-stress method [69] and the Voigt-Reuss-Hill approximation [70]. Based on the empirical Vickers hardness model $H_{\mathrm{v}}=2.0\left(k^{2} G\right)^{0.585}-3.0, k=G / B$, where $G$ and $B$ are the shear and bulk modulus [71], the calculated hardness values of $I 4 / \mathrm{mmm} \mathrm{B}_{4}$ and $\mathrm{Pm} \mathrm{B}_{17}$ are 27.3 and $26.8 \mathrm{GPa}$, respectively, indicating that they are good candidates as hard materials.

\section{Electronic and superconducting properties}

Much effort has been made to explore the superconductivity of B allotropes [72-75]. To be noted, two-dimensional 
(2D) boron allotropes become superconducting due their unique structure and inherent metallity $[72,73] . I 4 / \mathrm{mmm} \mathrm{B}_{4}$ and $\mathrm{Pm} \mathrm{B}_{17}$ allotropes are metallic at ambient pressure, as shown in the electronic band structures and PDOS [Fig. S9 and Figs. 3(c), 3(d), and 3(f)]. Similar to $I 4 / \mathrm{mmm} \mathrm{NaB}_{4}$ and $P m \mathrm{Na}_{2} \mathrm{~B}_{17}$, B $2 p$ in $I 4 / m m m \mathrm{~B}_{4}$ and $P m \mathrm{~B}_{17}$ allotropes has also a large contribution to their metallic character, with a strong hybridization between B $2 s$ and B $2 p$ orbitals [Figs. 3(d) and 3(f)], which plays a key role in stabilizing the covalent $\mathrm{B}$ networks. Additionally, there is a clear Fermi surface nesting in $I 4 / \mathrm{mmm} \mathrm{B}_{4}$ along $Z \rightarrow A$ and $M \rightarrow \Gamma$ directions [Fig. 3(e)], with flatter bands along $A \rightarrow M$ and $R \rightarrow X$ [Fig. 3(c)], which induces high electronic density of states near the Fermi level [Figs. 3(d) and 3(f)]. There appears van Hove singularity [Fig. 3(f)] in $P m \mathrm{Na}_{2} \mathrm{~B}_{17}$. On the other hand, I4/mmm $\mathrm{B}_{4}$ and $P m \mathrm{~B}_{17}$ allotropes contain the novel B structural units, as described above. As these features might support the superconducting transition in these compounds, we have estimated their superconducting critical temperatures $\left(T_{c}\right)$ using the Allen-Dynes modified McMillan equation [76-81] on the basis of Bardeen-Cooper-Schrieffer (BCS) [82] theory with adopting a typical Coulomb pseudopotential of $\mu^{*}=0.1$. The superconducting temperatures of $I 4 / \mathrm{mmm} \mathrm{B}_{4}$ and $P m \mathrm{~B}_{17}$ are predicted to be 19.8 and $15.4 \mathrm{~K}$ at ambient pressure, which are comparable to $2 \mathrm{D}$ B polymorphs (e.g., $18.7 \mathrm{~K}$ for $\beta_{12}$ [74], $24.7 \mathrm{~K}$ for $\chi_{3}$ [74], and $17.9 \mathrm{~K}$ for $2 \mathrm{D}$ boron layer [75]). The calculated electron-phonon coupling parameters $\lambda$ of $I 4 / \mathrm{mmm}_{4}$ and $P m \mathrm{~B}_{17}$ at $1 \mathrm{~atm}$ are 0.65 and 0.67 , respectively, comparable to 0.61 in $\mathrm{MgB}_{2}$ [83]. As clearly revealed in Fig. 3(g), the electron-phonon coupling (EPC) constant of $I 4 / \mathrm{mmm}$ $\mathrm{B}_{4}$ mainly arises from the contribution of low-frequency phonon modes below $23.9 \mathrm{THz}$, giving the dominant contribution $(97.8 \%)$ to the integral EPC constant $\lambda$. The same is observed in $P m \mathrm{~B}_{17}$, in which low-frequency vibrations below $27.1 \mathrm{THz}$ contribute $96.7 \%$ of the total $\lambda$ [Fig. 3(h)]. Therefore, the superconducting mechanism is quite simi- lar to that of the superconducting $\mathrm{Li}_{6} \mathrm{P}$ electride [84], but different from the high-frequency $\mathrm{H}$-derived vibrations of high- $T_{\mathrm{c}} \mathrm{H}_{3} \mathrm{~S}$ [7,12], $\mathrm{H}_{3} \mathrm{Se}$ [85], and $\mathrm{CaYH}_{12}$ [86] superconductors and intermediate-frequency $\mathrm{H}$-derived vibrations of $\mathrm{H}_{4} \mathrm{Te}$ [87].

\section{CONCLUSIONS}

In summary, four hitherto unknown Na-B compounds (i.e., $\mathrm{Na}_{3} \mathrm{~B}_{2}, \mathrm{Na}_{2} \mathrm{~B}_{3}, \mathrm{NaB}_{4}$, and $\mathrm{Na}_{2} \mathrm{~B}_{17}$ ) are identified with first-principle swarm-intelligence structural search calculations. The arrangements of $\mathrm{B}$ atoms in these compounds evolve sequentially with increasing the $\mathrm{B}$ content from onedimensional $\mathrm{B}$ linear chains to $\mathrm{B}_{6}$ octahedra, $\mathrm{B}_{7}$ pentahedra, and three-dimensional $\mathrm{B}$ frameworks. Removing $\mathrm{Na}$ atoms from I4/mmm $\mathrm{NaB}_{4}$ and $P m \mathrm{NaB}_{17}$, two metallic $I 4 / \mathrm{mmm}$ $\mathrm{B}_{4}$ and $P m \mathrm{~B}_{17}$ allotropes become very stable at ambient conditions. Besides being potential hard compounds, among the already known three-dimensional boron allotropes, they show the highest superconducting transition temperatures of 19.8 and $15.4 \mathrm{~K}$. Our work provides an effective way to get superconducting boron allotropes.

\section{ACKNOWLEDGMENTS}

The authors acknowledge the funding supports from the Natural Science Foundation of China under Grants No. 21573037, No. 21873017, No. 11704062, No. 11974208, and No. 51732003, the Postdoctoral Science Foundation of China under Grant No. 2013M541283, the Natural Science Foundation of Jilin Province (20190201231JC), the "111" Project (No. B13013), and the Fundamental Research Funds for the Central Universities (2412017QD006). The work was carried out at National Supercomputer Center in Tianjin, and the calculations were performed on TianHe-1 (A). A.B. acknowledges financial support from the Spanish Ministry of Economy and Competitiveness (FIS2016-76617-P).
[1] C. J. Pickard, I. Errea, and M. I. Eremets, Annu. Rev. Condens. Matter Phys. 11, 57 (2020).

[2] A. R. Oganov, C. J. Pickard, Q. Zhu, and R. J. Needs, Nat. Rev. Mater. 4, 331 (2019).

[3] E. Zurek and T. Bi, J. Chem. Phys. 150, 050901 (2019).

[4] D.-H. Lee, Annu. Rev. Condens. Matter Phys. 9, 261 (2018).

[5] L. Zhang, Y. Wang, J. Lv, and Y. Ma, Nat. Rev. Mater. 2, 17005 (2017).

[6] A. Jain, Y. Shin, and K. A. Persson, Nat. Rev. Mater. 1, 15004 (2016).

[7] A. P. Drozdov, M. I. Eremets, I. A. Troyan, V. Ksenofontov, and S. I. Shylin, Nature (London) 525, 73 (2015).

[8] A. P. Drozdov, P. P. Kong, V. S. Minkov, S. P. Besedin, M. A. Kuzovnikov, S. Mozaffari, L. Balicas, F. F. Balakirev, D. E. Graf, V. B. Prakapenka, E. Greenberg, D. A. Knyazev, M. Tkacz, and M. I. Eremets, Nature (London) 569, 528 (2019).

[9] M. Somayazulu, M. Ahart, A. K. Mishra, Z. M. Geballe, M. Baldini, Y. Meng, V. V. Struzhkin, and R. J. Hemley, Phys. Rev. Lett. 122, 027001 (2019).
[10] L. Gao, Y. Y. Xue, F. Chen, Q. Xiong, R. L. Meng, D. Ramirez, C. W. Chu, J. H. Eggert, and H. K. Mao, Phys. Rev. B 50, 4260 (1994).

[11] Y. Li, J. Hao, H. Liu, Y. Li, and Y. Ma, J. Chem. Phys. 140, 174712 (2014).

[12] D. Duan, Y. Liu, F. Tian, D. Li, X. Huang, Z. Zhao, H. Yu, B. Liu, W. Tian, and T. Cui, Sci. Rep. 4, 6968 (2014).

[13] H. Liu, I. I. Naumov, R. Hoffmann, N. W. Ashcroft, and R. J. Hemley, Proc. Natl. Acad. Sci. USA 114, 6990 (2017).

[14] Y. Sun, J. Lv, Y. Xie, H. Liu, and Y. Ma, Phys. Rev. Lett. 123, 097001 (2019).

[15] J. J. Hamlin, Nature (London) 569, 491 (2019).

[16] P. V Zinin, L. C. Ming, H. A. Ishii, R. Jia, T. Acosta, and E. Hellebrand, J. Appl. Phys. 111, 114905 (2012).

[17] T. Irifune, A. Kurio, S. Sakamoto, T. Inoue, and H. Sumiya, Nature (London) 421, 599 (2003).

[18] D. Y. Kim, S. Stefanoski, O. O. Kurakevych, and T. A. Strobel, Nat. Mater. 14, 169 (2014).

[19] H.-J. Sung, W. H. Han, I.-H. Lee, and K. J. Chang, Phys. Rev. Lett. 120, 157001 (2018). 
[20] B. Albert and H. Hillebrecht, Angew. Chemie Int. Ed. 48, 8640 (2009).

[21] T. Ogitsu, E. Schwegler, and G. Galli, Chem. Rev. 113, 3425 (2013).

[22] K. Shirai, Jpn. J. Appl. Phys. 56, 05FA06 (2017).

[23] A. R. Oganov, J. Chen, C. Gatti, Y. Ma, Y. Ma, C. W. Glass, Z. Liu, T. Yu, O. O. Kurakevych, and V. L. Solozhenko, Nature (London) 457, 863 (2009).

[24] X.-L. He, X. Shao, T. Chen, Y.-K. Tai, X.-J. Weng, Q. Chen, X. Dong, G. Gao, J. Sun, X.-F. Zhou, Y. Tian, and H.-T. Wang, Phys. Rev. B 99, 184111 (2019).

[25] M. I. Eremets, V. V Struzhkin, H. Mao, and R. J. Hemley, Science 293, 272 (2001).

[26] J. Zhao and J. P. Lu, Phys. Rev. B 66, 092101 (2002).

[27] U. Häussermann, S. I. Simak, R. Ahuja, and B. Johansson, Phys. Rev. Lett. 90, 065701 (2003).

[28] Y. Ma, J. S. Tse, D. D. Klug, and R. Ahuja, Phys. Rev. B 70, 214107 (2004).

[29] D. Li, K. Bao, F. Tian, X. Jin, D. Duan, Z. He, B. Liu, and T. Cui, RSC Adv. 4, 203 (2014).

[30] W. Zhang, A. R. Oganov, A. F. Goncharov, Q. Zhu, S. E. Boulfelfel, A. O. Lyakhov, E. Stavrou, M. Somayazulu, V. B. Prakapenka, and Z. Konôpková, Science 342, 1502 (2013).

[31] M. Miao, Nat. Chem. 5, 846 (2013).

[32] J. Lin, Z. Zhao, C. Liu, J. Zhang, X. Du, G. Yang, and Y. Ma, J. Am. Chem. Soc. 141, 5409 (2019).

[33] B. Albert, Angew. Chem. Int. Ed. 37, 1117 (1998).

[34] Y. Zhong, C.-H. Hu, D. Wang, and H.-Y. Zhou, J. Alloys Compd. 731, 323 (2018).

[35] X.-L. He, X. Dong, Q. S. Wu, Z. Zhao, Q. Zhu, A. R. Oganov, Y. Tian, D. Yu, X.-F. Zhou, and H.-T. Wang, Phys. Rev. B 97, 100102(R) (2018).

[36] Y. Wang, J. Lv, L. Zhu, and Y. Ma, Phys. Rev. B 82, 094116 (2010).

[37] Y. Wang, J. Lv, L. Zhu, and Y. Ma, Comput. Phys. Commun. 183, 2063 (2012).

[38] G. Kresse and J. Furthmüller, Phys. Rev. B 54, 11169 (1996).

[39] P. Hohenberg and W. Kohn, Phys. Rev. 136, B864 (1964).

[40] W. Kohn and L. J. Sham, Phys. Rev. 140, A1133 (1965).

[41] P. E. Blöchl, Phys. Rev. B 50, 17953 (1994).

[42] P. Blaha, K. Schwarz, P. Sorantin, and S. B. Trickey, Comput. Phys. Commun. 59, 399 (1990).

[43] J. P. Perdew, K. Burke, and M. Ernzerhof, Phys. Rev. Lett. 77, 3865 (1996).

[44] J. P. Perdew, J. A. Chevary, S. H. Vosko, K. A. Jackson, M. R. Pederson, D. J. Singh, and C. Fiolhais, Phys. Rev. B 46, 6671 (1992).

[45] H. J. Monkhorst and J. D. Pack, Phys. Rev. B 13, 5188 (1976).

[46] P. Giannozzi, S. Baroni, N. Bonini, M. Calandra, R. Car, C. Cavazzoni, D. Ceresoli, G. L. Chiarotti, M. Cococcioni, I. Dabo, A. Dal Corso, S. de Gironcoli, S. Fabris, G. Fratesi, R. Gebauer, U. Gerstmann, C. Gougoussis, A. Kokalj, M. Lazzeri, L. Martin-Samos, N. Marzari, F. Mauri, R. Mazzarello, S. Paolini, A. Pasquarello, L. Paulatto, C. Sbraccia, S. Scandolo, G. Sclauzero, A. P. Seitsonen, A. Smogunov, P. Umari, and R. M. Wentzcovitch, J. Phys. Condens. Matter 21, 395502 (2009).

[47] See Supplemental Material at http://link.aps.org/supplemental/ 10.1103/PhysRevB.101.174507 for computational details. Phonon dispersion curves, projected phonon density of states (PHDOS), electronic band structures, projected density of states
(PDOS), and electron localization function (ELF) of predicted stable Na-B phases. ELF, mechanical, and superconducting properties of $I 4 / \mathrm{mmm} \mathrm{B}_{4}$ and $P m \mathrm{~B}_{17}$.

[48] J. Feng, R. G. Hennig, N. W. Ashcroft, and R. Hoffmann, Nature (London) 451, 445 (2008).

[49] C. S. Barrett, Acta Crystallogr. 9, 671 (1956).

[50] M. Hanfland, I. Loa, and K. Syassen, Phys. Rev. B 65, 184109 (2002).

[51] F. Peng, M. Miao, H. Wang, Q. Li, and Y. Ma, J. Am. Chem. Soc. 134, 18599 (2012).

[52] C.-H. Hu, A. R. Oganov, Q. Zhu, G.-R. Qian, G. Frapper, A. O. Lyakhov, and H.-Y. Zhou, Phys. Rev. Lett. 110, 165504 (2013).

[53] S. Zhang, J. Lin, Y. Wang, G. Yang, A. Bergara, and Y. Ma, J. Phys. Chem. C 122, 12022 (2018).

[54] K. Parlinski, Z. Q. Li, and Y. Kawazoe, Phys. Rev. Lett. 78, 4063 (1997).

[55] A. Togo, F. Oba, and I. Tanaka, Phys. Rev. B 78, 134106 (2008).

[56] A. Hermann, A. McSorley, N. W. Ashcroft, and R. Hoffmann, J. Am. Chem. Soc. 134, 18606 (2012).

[57] A. D. Becke and K. E. Edgecombe, J. Chem. Phys. 92, 5397 (1990).

[58] R. Dronskowski and P. E. Bloechl, J. Phys. Chem. 97, 8617 (1993).

[59] S. Maintz, V. L. Deringer, A. L. Tchougréeff, and R. Dronskowski, J. Comput. Chem. 37, 1030 (2016).

[60] Z. J. Wu, E. J. Zhao, H. P. Xiang, X. F. Hao, X. J. Liu, and J. Meng, Phys. Rev. B 76, 054115 (2007).

[61] G. J. Martyna, M. L. Klein, and M. Tuckerman, J. Chem. Phys. 97, 2635 (1992).

[62] C. Fan, J. Li, and L. Wang, Sci. Rep. 4, 6786 (2014).

[63] M. J. van Setten, M. A. Uijttewaal, G. A. de Wijs, and R. A. de Groot, J. Am. Chem. Soc. 129, 2458 (2007).

[64] Q. An, K. Madhav Reddy, K. Y. Xie, K. J. Hemker, and W. A. Goddard, Phys. Rev. Lett. 118, 159902(E) (2017).

[65] H.-Y. Chung, M. B. Weinberger, J. B. Levine, A. Kavner, J.-M. Yang, S. H. Tolbert, and R. B. Kaner, Science 316, 436 (2007).

[66] Q. Gu, G. Krauss, and W. Steurer, Adv. Mater. 20, 3620 (2008).

[67] R. Mohammadi, A. T. Lech, M. Xie, B. E. Weaver, M. T. Yeung, S. H. Tolbert, and R. B. Kaner, Proc. Natl. Acad. Sci. USA 108, 10958 (2011).

[68] H. Gou, N. Dubrovinskaia, E. Bykova, A. A. Tsirlin, D. Kasinathan, W. Schnelle, A. Richter, M. Merlini, M. Hanfland, A. M. Abakumov, D. Batuk, G. Van Tendeloo, Y. Nakajima, A. N. Kolmogorov, and L. Dubrovinsky, Phys. Rev. Lett. 111, 157002 (2013).

[69] Y. Le Page and P. Saxe, Phys. Rev. B 65, 104104 (2002).

[70] R. Hill, Proc. Phys. Soc. Sect. A 65, 349 (1952).

[71] X.-Q. Chen, H. Niu, D. Li, and Y. Li, Intermetallics 19, 1275 (2011).

[72] E. S. Penev, A. Kutana, and B. I. Yakobson, Nano Lett. 16, 2522 (2016).

[73] Y. Zhao, S. Zeng, and J. Ni, Phys. Rev. B 93, 014502 (2016).

[74] M. Gao, Q.-Z. Li, X.-W. Yan, and J. Wang, Phys. Rev. B 95, 024505 (2017).

[75] T. Chen, Q. Gu, Q. Chen, X. Wang, C. J. Pickard, R. J. Needs, D. Xing, and J. Sun, Phys. Rev. B 101, 054518 (2020).

[76] W. L. McMillan, Phys. Rev. 167, 331 (1968).

[77] P. B. Allen and R. C. Dynes, Phys. Rev. B 12, 905 (1975). 
[78] J. P. Carbotte, Rev. Mod. Phys. 62, 1027 (1990).

[79] L. N. Oliveira, E. K. U. Gross, and W. Kohn, Phys. Rev. Lett. 60, 2430 (1988).

[80] M. Lüders, M. A. L. Marques, N. N. Lathiotakis, A. Floris, G. Profeta, L. Fast, A. Continenza, S. Massidda, and E. K. U. Gross, Phys. Rev. B 72, 024545 (2005).

[81] M. A. L. Marques, M. Lüders, N. N. Lathiotakis, G. Profeta, A. Floris, L. Fast, A. Continenza, E. K. U. Gross, and S. Massidda, Phys. Rev. B 72, 024546 (2005).

[82] J. Bardeen, L. N. Cooper, and J. R. Schrieffer, Phys. Rev. 108, 1175 (1957).
[83] H. J. Choi, D. Roundy, H. Sun, M. L. Cohen, and S. G. Louie, Phys. Rev. B 66, 020513(R) (2002).

[84] Z. Zhao, S. Zhang, T. Yu, H. Xu, A. Bergara, and G. Yang, Phys, Rev. Lett. 122, 097002 (2019).

[85] S. Zhang, Y. Wang, J. Zhang, H. Liu, X. Zhong, H.-F. Song, G. Yang, L. Zhang, and Y. Ma, Sci. Rep. 5, 15433 (2015).

[86] X. Liang, A. Bergara, L. Wang, B. Wen, Z. Zhao, X.-F. Zhou, J. He, G. Gao, and Y. Tian, Phys. Rev. B 99, 100505(R) (2019).

[87] X. Zhong, H. Wang, J. Zhang, H. Liu, S. Zhang, H.-F. Song, G. Yang, L. Zhang, and Y. Ma, Phys. Rev. Lett. 116, 057002 (2016). 